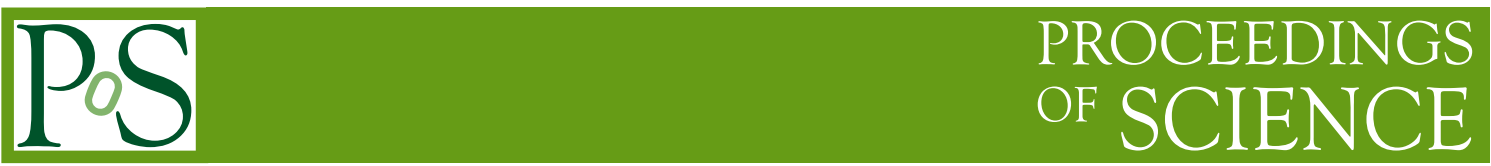

\title{
Measurement of Liquid Scintillator Nonlinearity
}

\section{Tadeáš Dohnal, ${ }^{a *}$ Vít Vorobel $^{a}$ and Tomáš Tměj ${ }^{a}$}

${ }^{a}$ Institute of Particle and Nuclear Physics, Faculty of Mathematics and Physics, Charles University V Holešovičkách 2, 18000 Prague 8, Czech Republic

E-mail: dohnal@ipnp.mff.cuni.cz, vorobel@ipnp.mff.cuni.cz, tmej@ipnp.mff.cuni.cz

Organic liquid scintillator (LS) is a common choice for detectors precisely measuring energy of electron antineutrinos, such as JUNO currently under construction. Accurate knowledge of the relation between the scintillation light response and the energy deposited by a particle is essential for determination of the antineutrino energy. The response is not exactly linear. Deviation from the LS linearity is the subject of the presented investigation. The method of measurement is using Compton scattering of gammas of well known energy in the LS and precise measurement of the scattered gamma energy with a HPGe detector.

40th International Conference on High Energy physics - ICHEP2020

July 28 - August 6, 2020

Prague, Czech Republic (virtual meeting)

${ }^{*}$ Speaker 


\section{Organic Liquid Scintillators}

Organinic liquid scintillators (LS) are hydrocarbon compounds that contain benzene-ring structures. When scintillator molecules are excited by an ionizing particle, fraction of the energy is emitted as light that can be detected by a photomultiplier tube (PMT) or other light-detecting device. It is commonly used by experiments that measure oscillation of reactor antineutrinos, such as the experiment JUNO [1] currently under construction. In these experiments, the antineutrinos typically interact via inverse beta decay: $\bar{v}_{e}+p \rightarrow e^{+}+n$. Energy of $\bar{v}_{e}$ can be deduced from the $e^{+}$energy. However, the dependence of the amount of scintillation light on the deposited energy is not exactly linear. Knowledge of this dependence is important for the $\bar{v}_{e}$ energy reconstruction.

\section{LS Nonlinearity}

The deviation from linear dependence of the amount of scintillation light on the deposited energy is caused by quenching interactions between excited molecules along the path of the incident particle, i.e., interactions that drain the energy which would otherwise go into luminescence. Since a higher ionizing power produces a higher density of excited molecules, more quenching interactions will take place for these particles. This effect is described by a semi-empirical Birk's formula [2]:

$$
\frac{d L}{d x} \propto \frac{\frac{d E}{d x}}{1+k_{\mathrm{B}} \frac{d E}{d x}},
$$

where $\frac{d L}{d x}$ is the light output per unit length, $\frac{d E}{d x}$ is the energy loss per unit length and $k_{\mathrm{B}}$ is the Birk's constant.

\section{Experimental Principle and Setup}

In our setup (see Fig. 1), $\gamma$ source emits mono-energetic photons. If such photon interacts via Compton scattering in the LS, it transfers part of its energy to an electron. The recoiled electron causes production of scintillation light which is detected by a PMT. It is affected by quenching.

Meanwhile, the energy of the scattered photon is precisely measured by a HPGe detector. Thus the energy deposited in the LS can be determined. Signal from the LS is then compared to the energy determined by the HPGe detector. Due to the Compton scattering kinematics, varying the angle $\theta$ enables us to scan various energies.

Four radioisotopes $\left({ }^{137} \mathrm{Cs},{ }^{22} \mathrm{Na},{ }^{60} \mathrm{Co},{ }^{24} \mathrm{Na}\right)$ were used along with ${ }^{241} \mathrm{Am}$ for monitoring of the LS energy scale stability. The LS based on linear alkylbenzene was placed inside a cylindrical vessel with bottom attached to a 3" PMT and sides made of a reflective surface (PTFE).

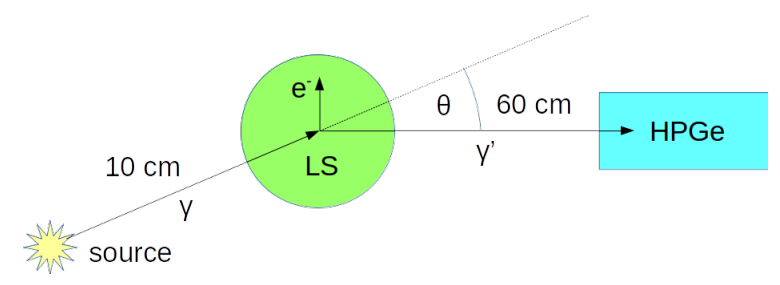

Figure 1: Scheme of the experimental setup. 


\section{Energy Scale Stability \& Readout Nonlinearity}

The HPGe was calibrated using 8 radioisotopes with the difference between reconstructed and table values within $0.04 \mathrm{keV}$. The HPGe energy scale was stable within $0.1 \%$.

The LS \& PMT readout energy scale instability was in the order of ones of \%. For that reason, corrections based on the position of the Compton edge and the ${ }^{241} \mathrm{Am}$ peak were applied.

PMT readout nonlinearity was measured using two LEDs triggered by the same pulse generator. The signal of both LEDs flashing simultaneously was compared to the sum of the signals of LEDs flashing individually. The deviation from linear response was within 1\%; correction was applied.

\section{LS Nonlinearity Data Analysis}

First, the LS is linearly 'pre-calibrated' at a certain energy using data from the HPGe. Coincidence events are transformed from $E_{\text {scint }}$ vs $E_{\mathrm{HPGe}}$ to $E_{\mathrm{scint}}+E_{\mathrm{HPGe}}$ vs $E_{\mathrm{HPGe}}$. Projection to $E_{\mathrm{scint}}+E_{\mathrm{HPGe}}$ axis is made for various bins in $E_{\mathrm{HPGe}}$ and the peaks are fitted. $E_{\text {scint }}$ is extracted from the fitted value and compared to $E_{\gamma}-E_{\mathrm{HPGe}}$.

The LS nonlinearity was observed in several samples. Ideally, data points obtained with the apparatus set to various angles $\theta$ should partially overlap creating nonlinearity curve (see Fig. 2a), especially for measurements done at $30^{\circ}$ and $-30^{\circ}$ (i.e. $30^{\circ}$ angle set in opposite direction - see Fig. 2b). Unfortunately, this was not exactly the case as there are detector-induced effects present that make precise deduction of $k_{\mathrm{B}}$ difficult: PMT offset, light collection and PMT efficiency nonuniformities. These issues need to be carefully addressed before final results are determined.

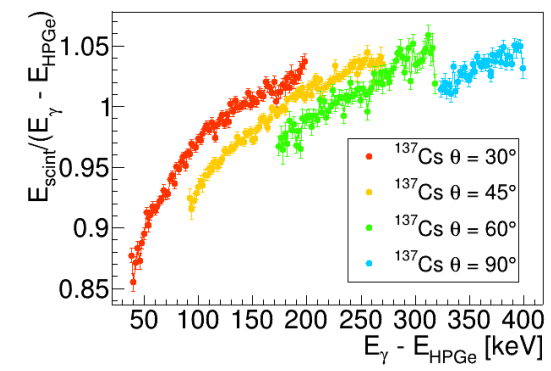

(a) Whole energy range

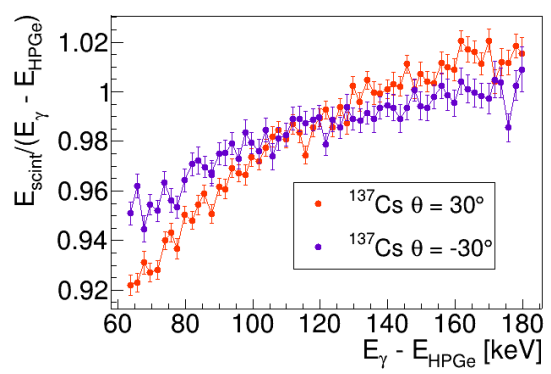

(b) Detail for mirrored geometry

Figure 2: Measured LS nonlinearity curve

\section{Acknowledgments}

This work was supported in part by the Charles University GAUK Project No. 284317 and Grant MŠMT ČR, Inter-Excelence, LTT17021.

\section{References}

[1] T. Adam et al., arXiv:1508.07166 [physics.ins-det] (2015)

[2] W. R. Leo, Techniques for Nuclear and Particle Physics Experiments. Springer-Verlag (1987) 